\title{
Casimir-Polder-like force on an atom outside a Schwarzschild black hole
}

\author{
Jialin Zhang ${ }^{1}$ and Hongwei $\mathrm{Yu}^{1,2}$ \\ 1 Institute of Physics and Key Laboratory of Low Dimensional \\ Quantum Structures and Quantum Control of Ministry of Education, \\ Hunan Normal University, Changsha, Hunan 410081, China \\ 2 Center for Nonlinear Science and Department of Physics, \\ Ningbo University, Ningbo, Zhejiang 315211, China
}

\begin{abstract}
We calculate, in the framework of open quantum systems, the ground state energy-level shift for a static two-level atom outside a spherically symmetric black hole in interaction with fluctuating massless scalar fields in the Boulware and Unruh vacuums. We find that the energy-level shift is position dependent and thus gives rise to a force on the atom besides the classical gravitational force. For the case of the Boulware vacuum that represents a star which has not collapsed through its event horizon, this force is attractive near the horizon and is repulsive far away from the black hole with a behavior of $r^{-3}$. For the case of the Unruh vacuum which represents a radiating black hole, we find that the contribution to the Casimir-Polder-like force due to the presence of Hawking radiation is always attractive and, remarkably, this attractive force diverges at the event horizon.
\end{abstract}

PACS numbers: 12.20.Ds, 03.65.Yz, 04.62.+v, 04.70.Dy 


\section{INTRODUCTION}

The shift of the energy-levels of an atom which is caused by the coupling of the atom with the quantum vacuum is one of the striking manifestations of the existence of zero-point fluctuations, and it is known that this shift can be modified by the presence of cavities [1] and the non-inertial motion of the atom itself [2 [5]. One of the observable examples of such shifts is the Casimir-Polder force between a neutral electric polarizable atom and a conducting plate, which is a result of position-dependent energy-level shift caused by the modification of vacuum fluctuations that arise because of reflection of vacuum field modes at the conducting boundaries.

So far, the Casimir-Polder force, which is a consequence of the position-dependent level shifts of an atom, is regarded as a result of the reshaping of vacuum fluctuations induced by the reflection of field modes at boundaries in flat spacetimes. Since the vacuum field modes are also scattered by the curvature in a curved spacetime, such as in the background of a black hole, a question naturally arises as to whether the scattering of vacuum field modes off the curvature will also produce a Casimir-Polder-like force on an atom outside a back hole, and this is exactly what we are going to address in the present paper.

Our calculation of the energy-level shifts is based upon the framework of open quantum systems [6], where a static two-level atom outside a Schwarzschild back hole is treated as an open quantum system in interaction with the external reservoir of fluctuating vacuum massless scalar fields. As for any open system, the full dynamics of the atom can be obtained from the complete time evolution describing the total system (atom plus a reservoir of external vacuum fields) by integrating over the field degrees of freedom, which are in fact not observed. It is worth noting here that the open quantum system approach has also been applied to studying other quantum effects in curved spacetime, such as the Hawking radiation of a black hole [7] and the Gibbons-Hawking effect of de Sitter spacetime [8].

When a curved space-time is considered as opposed to a flat one, a delicate issue arises as to how the vacuum state of the quantum fields is specified. In this paper, we deal with two vacuum states of the scalar fields; namely, the Boulware vacuum and the Unruh vacuum. Let 
us note that the Boulware vacuum would be the vacuum state outside a massive spherical body of radius only slightly larger than its Schwarzschild radius and the Unruh state that best approximates the vacuum following the gravitational collapse of a massive body to a black hole. So, we are going to compute the force on a static atom as a result of the modified vacuum fluctuations both outside a star which has not collapsed through its event horizon and a black hole.

The paper is organized as follows: In the next section, we will review the basic formalism of open quantum systems, the derivation of the master equation describing the system of the atom plus external vacuum scalar fields in the weak-coupling limit, and the reduced dynamics it generates for the finite-time evolution of the atom. In Sec. III, we calculate the radiative energy shift of the ground state and the resulting force on the atom. Finally, we conclude in Section IV.

\section{THE BASIC FORMALISM}

We consider the evolution in the proper time of a static two-level atom interacting with vacuum massless scalar fields outside a Schwarzschild black hole and assume the combined system (atom + external vacuum fields) to be initially prepared in a factorized state, with the atom held static in the exterior region of the black hole and the fields in the Boulware or Unruh vacuum state. When the quantum system of a two-level atom interacts weakly with the environment, the reduced dynamics can be obtained by eliminating the environment degrees of freedom, yielding an evolution equation of the atom that satisfies the master equation $[9$ 11]. We take the total Hamiltonian for the complete system to have the form

$$
H=H_{S}+H_{\phi}+H_{I}
$$

where $H_{S}$ denotes the free Hamiltonian of the atom and $H_{\phi}$ that of the environment(a bath of fluctuating quantum fields). In fact, $H_{\phi}$ can be chosen as the standard Hamiltonian of massless, free scalar fields, the details of which are not relevant here. In order to make our discussion generic, we will postpone the specification of $H_{S}$ until later and suppose that the 
interaction Hamiltonian has the general form

$$
H_{I}=\mu \sum_{\alpha} A_{\alpha} \otimes B_{\alpha}
$$

where $A_{\alpha}$ and $B_{\alpha}$ represent respectively the dynamical variables of the atom and of the environment. It should be pointed out that the coupling constant $\mu$ should be small, and this is required by our assumption that the interaction of the atom with the scalar fields is weak.

Initially, the complete system is described by the total density $\rho_{\text {tot }}=\rho(0) \otimes \rho_{B}$, where $\rho(0)$ is the initial reduced density matrix of the atom, and $\rho_{B}$ is the state of the environment. In the frame of the atom, the evolution in the proper time $\tau$ of the total density matrix $\rho_{t o t}$ of the complete system satisfies

$$
\frac{\partial \rho_{t o t}(\tau)}{\partial \tau}=-i L_{H}\left[\rho_{t o t}(\tau)\right]
$$

where the symbol $L_{H}$ represents the Liouville operator associated with $H$

$$
L_{H}[S] \equiv[H, S]
$$

The dynamics of the atom can be obtained by tracing over the field degrees of freedom, that is, by applying the trace projection to the total density matrix $\rho(\tau)=\operatorname{Tr}_{\Phi}\left[\rho_{\text {tot }}(\tau)\right]$.

In the limit of weak-coupling which we assume in the present paper, the reduced density is found to obey the master equation in the interaction picture [6, 12$]$

$$
\frac{d}{d \tau} \rho_{S}(\tau)=-i\left[H_{L S}, \rho_{S}(\tau)\right]+\mathcal{D}\left(\rho_{S}(\tau)\right)
$$

where $H_{L S}$ provides a Hamiltonian contribution to the dynamics of the system and is the so-called Lamb shift Hamiltonian, whereas, $\mathcal{D}\left(\rho_{S}(\tau)\right)$ is called the dissipator of the master equation. Let us note that here $H_{L S}$ is given by

$$
H_{L S}=\mu^{2} \sum_{\omega} \sum_{\alpha, \beta} S_{\alpha \beta}(\omega) A_{\alpha}^{+}(\omega) A_{\beta}(\omega)
$$

and the system operator can be decomposed as [6]

$$
A_{\alpha}=\sum_{\omega} A_{\alpha}(\omega)=\sum_{\omega} A_{\alpha}^{+}(\omega)
$$


with

$$
A_{\alpha}(\omega)=\sum_{\nu^{\prime}-\nu=\omega} \Pi(\nu) A_{\alpha} \Pi\left(\nu^{\prime}\right),
$$

where the operator $\Pi(\nu)$ denotes the projection onto the eigenspace belonging to the eigenvalue $\nu$ of $H_{S}$. It is easy to see that

$$
\left[H_{S}, A_{\alpha}^{+}(\omega) A_{\beta}(\omega)\right]=0
$$

that is, $H_{L S}$ has the same eigenstates as $H_{S}$. Meanwhile, the function $S_{\alpha \beta}(\omega)$ can be written as [6]

$$
S_{\alpha \beta}(\omega)=\frac{i}{2} \mathcal{G}_{\alpha \beta}(\omega)-i \Gamma_{\alpha \beta}(\omega),
$$

where $\mathcal{G}_{\alpha \beta}(\omega)$ is the Fourier transform of the reservoir correlation function $\left(\left\langle B_{\alpha}^{+}(s) B_{\beta}(0)\right\rangle\right)$

$$
\mathcal{G}_{\alpha \beta}(\omega)=\int_{-\infty}^{\infty} d s e^{i \omega s}\left\langle B_{\alpha}^{+}(s) B_{\beta}(0)\right\rangle
$$

and $\Gamma_{\alpha \beta}(\omega)$ denotes the one-side Fourier transform

$$
\Gamma_{\alpha \beta}(\omega)=\int_{0}^{\infty} d s e^{i \omega s}\left\langle B_{\alpha}^{+}(s) B_{\beta}(0)\right\rangle .
$$

Then, with the help of

$$
\frac{1}{1 \mp i \epsilon}=P \frac{1}{x} \pm i \pi \delta(x)
$$

where $\mathrm{P}$ denotes the Cauchy principal value, it is easy to prove that

$$
S_{\alpha \beta}(\omega)=-\frac{P}{2 \pi} \int_{-\infty}^{\infty} \frac{\mathcal{G}_{\alpha \beta}(z)}{z-\omega} d z
$$

The dissipator of the master equation takes the form

$$
\mathcal{D}\left(\rho_{S}\right)=\mu^{2} \sum_{\omega} \sum_{\alpha, \beta} \mathcal{G}_{\alpha \beta}(\omega)\left(A_{\beta}(\omega) \rho_{S} A_{\alpha}^{+}(\omega)-\frac{1}{2}\left\{A_{\alpha}^{+}(\omega) A_{\beta}(\omega), \rho_{S}\right\}\right) .
$$

The Hamiltonian $H_{S}$ of a two-level atom can be generically written as [10]

$$
H_{S}=\frac{\omega_{0}}{2} \sum_{i=1}^{3} n_{i} \sigma_{i},
$$


where $\sigma_{i}(i=1,2,3)$ are the Pauli matrices, $\omega_{0}$ is the energy-level spacing and $\mathbf{n}=\left(n_{1}, n_{2}, n_{3}\right)$ is a unit vector. Now, we let $\mathbf{n}=(0,0,1)$ (i.e., $\left.H_{S}=\omega_{0} \sigma_{3} / 2\right)$ for simplicity. The interaction Hamiltonian $H_{I}$ is taken as

$$
H_{I}=\mu \sum_{\alpha=0}^{3} \sigma_{\alpha} \Phi_{\alpha}(x)
$$

where $\sigma_{0}$ is the unit matrix and external fields are represented by $\Phi_{\alpha}(x)$. Comparing it with the general form Eq. (2), we find that $A_{\alpha}=\sigma_{\alpha}, B_{\alpha}=\Phi_{\alpha}(x)$.

For the sake of simplicity, we now assume that the field correlation functions are diagonal such that [10]

$$
G^{+}(x-y) \delta_{\alpha \beta}=\left\langle\Phi_{\alpha}(x) \Phi_{\beta}(y)\right\rangle
$$

Therefore, its Fourier and Hilbert transforms can be expressed respectively as

$$
\mathcal{G}(\omega)=\int_{-\infty}^{\infty} d \tau e^{i \omega \tau} G^{+}(x(\tau))=\delta_{\alpha \beta} \mathcal{G}_{\alpha \beta}(\omega)
$$

and

$$
\mathcal{K}(\omega)=\frac{P}{\pi i} \int_{-\infty}^{\infty} d \lambda \frac{\mathcal{G}(\lambda)}{\lambda-\omega}=2 i \delta_{\alpha \beta} S_{\alpha \beta}(\omega) .
$$

It should be pointed out that here the repeated index does not mean summation. Letting $|+\rangle$ and $|-\rangle$ denote the excited and the ground states, respectively, we have

$$
A_{\alpha}\left(\omega_{0}\right)=A_{\alpha}^{+}\left(-\omega_{0}\right)=|-\rangle\left\langle-\left|\sigma_{\alpha}\right|+\right\rangle\langle+|
$$

Since the energy spacing is $\omega_{0}$, according to Eq. (8), the summation over $\omega$ in Eq. (6) just contains two terms; namely, $\omega=\omega_{0}$ and $\omega=-\omega_{0}$. As a result, Eq. (6) can be rewritten as

$$
\begin{aligned}
H_{L S} & =\mu^{2} \sum_{\omega} \sum_{\alpha, \beta} S_{\alpha \beta}(\omega) A_{\alpha}^{+}(\omega) A_{\beta}(\omega) \\
& =-i \frac{\mu^{2}}{2} \mathcal{K}\left(\omega_{0}\right) \sum_{\alpha=0}^{3}|+\rangle\left|\left\langle+\left|\sigma_{\alpha}\right|-\right\rangle\right|^{2}\left\langle+\left|-i \frac{\mu^{2}}{2} \mathcal{K}\left(-\omega_{0}\right) \sum_{\alpha=0}^{3}\right|-\right\rangle\left|\left\langle-\left|\sigma_{\alpha}\right|+\right\rangle\right|^{2}\langle-| \\
& =-i \mu^{2} \mathcal{K}\left(\omega_{0}\right)|+\rangle\left\langle+\left|-i \mu^{2} \mathcal{K}\left(-\omega_{0}\right)\right|-\right\rangle\langle-|
\end{aligned}
$$

This shows that the energy-level shifts of the ground state and excited state are $\delta E_{-}=$ $-i \mu^{2} \mathcal{K}\left(-\omega_{0}\right)$ and $\delta E_{+}=-i \mu^{2} \mathcal{K}\left(\omega_{0}\right)$, respectively, which are caused by the coupling of the 
atom to the vacuum fluctuation of the fields. The relative energy shift (the Lamb shift) is then

$$
\Delta=i \mu^{2}\left[\mathcal{K}\left(-\omega_{0}\right)-\mathcal{K}\left(\omega_{0}\right)\right]
$$

\section{CASIMIR-POLDER-LIKE FORCE FOR BOULWARE VACUUM}

In this section, we apply the open quantum system formalism developed in the preceding section to address the issue of finite-time evolution of a static two-level atom interacting with vacuum scalar fields outside a spherically symmetric black hole and calculate the energy-level shift of the ground state. The line element for a Schwarzschild black hole reads

$$
d s^{2}=g_{\mu \nu} x^{\mu} x^{\nu}=\left(1-\frac{2 M}{r}\right) d t^{2}-\frac{d r^{2}}{1-2 M / r}-r^{2}\left(d \theta^{2}+\sin ^{2} \theta d \phi^{2}\right) .
$$

Let us start with the Boulware vacuum which is deemed to be the natural vacuum outside a massive body and reduces to the usual Minkowski vacuum at infinity. The correlation function for the scalar field in the Boulware vacuum is [13, 14]

$$
G_{B}^{+}\left(x, x^{\prime}\right)=\sum_{l m} \int_{0}^{\infty} \frac{e^{-i \omega \Delta t}}{4 \pi \omega}\left|Y_{l m}(\theta, \phi)\right|^{2}\left[\left|\overrightarrow{R_{l}}(\omega, r)\right|^{2}+\left|\overleftarrow{R_{l}}(\omega, r)\right|^{2}\right] d \omega
$$

and the corresponding Fourier transform with the proper time $\tau$ of the atom reads

$$
\begin{aligned}
\mathcal{G}_{B}(\lambda) & =\int_{-\infty}^{\infty} e^{i \lambda \tau} G_{B}^{+}[x(\tau)] d \tau \\
& =\sum_{m l} \int_{0}^{\infty} \frac{d \omega}{2 \omega} \delta(\lambda-\omega / \sqrt{1-2 M / r})\left|Y_{l m}(\theta, \phi)\right|^{2}\left[\left|\overrightarrow{R_{l}}(\omega, r)\right|^{2}+\left|\overleftarrow{R_{l}}(\omega, r)\right|^{2}\right]
\end{aligned}
$$

Using Eq. (20), we can calculate $\delta E_{-}$for the Boulware vacuum; for convenience, we denote it by $\delta E_{-}^{B}$. Since the exact form of functions $R_{l}(\omega, r)$ is not known, a generic expression for $\delta E_{-}^{B}$ is not possible to obtain. In what follows, we only examine the behavior of $\delta E_{-}^{B}$ at two asymptotic regions, i.e., close to the black hole horizon and at infinity. In order to do this, let us recall that [14]

$$
\sum_{l=0}^{\infty}(2 l+1)\left|\vec{R}_{l}(\omega, r)\right|^{2} \sim \begin{cases}\frac{4 \omega^{2}}{1-\frac{2 M}{r}}, & r \rightarrow 2 M \\ \frac{1}{r^{2}} \sum_{l=0}^{\infty}(2 l+1)\left|B_{\ell}(\omega)\right|^{2}, & r \rightarrow \infty\end{cases}
$$




$$
\sum_{l=0}^{\infty}(2 l+1)\left|\overleftarrow{R}_{l}(\omega, r)\right|^{2} \sim \begin{cases}\frac{1}{r^{2}} \sum_{\ell=0}^{\infty}(2 l+1)\left|B_{\ell}(\omega)\right|^{2}, & r \rightarrow 2 M \\ 4 \omega^{2} /(1-2 M / r) \sim 4 \omega^{2}, & r \rightarrow \infty\end{cases}
$$

Here $B_{\ell}$ is the transmission amplitude. The energy-level shift $\delta E_{-}^{B}$ in two special cases can then be calculated. We find, both when $r \rightarrow 2 M$ and $r \rightarrow \infty$, that

$$
\delta E_{-}^{B}=-i \mu^{2} \mathcal{K}_{B}\left(-\omega_{0}\right) \approx \delta E_{0}+\delta E_{0 r}
$$

Here, we have defined

$$
\begin{gathered}
\delta E_{0}=-\frac{\mu^{2} P}{2 \pi^{2}} \int_{0}^{\infty} \frac{\lambda}{\lambda+\omega_{0}} d \lambda, \\
\delta E_{0 r}=-\frac{\mu^{2} P}{2 \pi^{2}} \int_{0}^{\infty} f(\lambda, r) \frac{\lambda}{\lambda+\omega_{0}} d \lambda,
\end{gathered}
$$

where

$$
f(\lambda, r)=\sum_{\ell=0}^{\infty} \frac{(1+2 l)\left|B_{\ell}\left(\lambda \sqrt{g_{00}}\right)\right|^{2}}{4 \lambda^{2} r^{2}}
$$

and $P$ denotes the principal value. For the purpose of estimating $\delta E_{-}^{B}$ approximately, we use the geometrical optics approximation to evaluate the transmission amplitude $B_{\ell}(p)$ [15]. In this approximation, it is easy to verify that, if $M p \gg 1$, then transmission ceases when $l$ exceeds $\sqrt{27} M p$ and this holds even when $M p$ is small [15]. So we have

$$
B_{\ell}(p) \sim \theta(\sqrt{27} M p-l), \sum_{\ell}(1+2 l)\left|B_{\ell}(p)\right|^{2} \approx 27 M^{2} p^{2}
$$

This leads to

$$
f(\lambda, r) \approx \frac{27 M^{2} g_{00}}{4 r^{2}}
$$

Therefore, we can rewrite $\delta E_{-}^{B}$ in both two special cases as

$$
\delta E_{-}^{B} \approx \delta E_{0}+\frac{27 M^{2} g_{00}}{4 r^{2}} \delta E_{0}
$$

Here, $\delta E_{0}$ is just the shift of the ground state energy level in the Minkowski vacuum[2]. So, $\delta E_{-}^{B}$ contains two divergent terms, both of which contain a linearly divergent factor $\delta E_{0}$. 
According to the Bethe's mass renormalization method[16], $\delta E_{0}$ can be written in two parts

$$
\delta E_{0}=\delta E^{\prime}{ }_{0}+\delta E^{\prime \prime}{ }_{0}=\frac{-\mu^{2} P}{2 \pi^{2}} \int_{0}^{\infty} d \lambda+\frac{\mu^{2} P}{2 \pi^{2}} \int_{0}^{\infty} \frac{\omega_{0}}{\lambda+\omega_{0}} d \lambda,
$$

where $\delta E^{\prime}{ }_{0}$ is the energy of a free electron due to its coupling to the field, which can also be interpreted as the element stemming from the renormalization of the mass in the kinetic energy of the system Hamiltonian. However, it will not contribute to the observed level shift. The second part

$$
\delta E^{\prime \prime}{ }_{0}=\frac{\mu^{2} P}{2 \pi^{2}} \int_{0}^{\infty} \frac{\omega_{0}}{\lambda+\omega_{0}} d \lambda
$$

although logarithmically divergent, will give an observable contribution to the atomic level shift after a regularization by taking a cutoff of the upper limit of integration. For the regularization, we assume that the Bethe method [16] which was first used in a flat spacetime can also be applied in the present case. According to Bethe, this cutoff should be taken as the electron mass $m^{1}$. Therefore, the renormalized energy-level shift $\delta E_{-}^{B}$ reads

$$
\delta E_{-}^{B} \approx \frac{\mu^{2} \omega_{0}}{2 \pi^{2}} \ln \left(\frac{m}{\omega_{0}}\right)\left[1+\frac{27 M^{2} g_{00}}{4 r^{2}}\right]
$$

It is easy to see that the regular finite energy-level shift is position-dependent, and so generates a force on the atom besides the gravitational force. This is a force which has a quantum origin and it is in fact a result of the modified vacuum fluctuations due to the spacetime curvature. This position-dependent energy shift gives rise to a force on the atom which can be calculated by taking the first derivative with respect to $r$. Therefore, we can obtain the Casimir-Polder-like force on an atom outside a spherical star in two asymptotic regions (i.e., $r \rightarrow \infty$ and $r \rightarrow 2 M):$

$$
F^{B}=-\frac{\partial\left(\delta E_{-}^{B}\right)}{\partial r} \approx \frac{27 \mu^{2} M^{2} \omega_{0}}{4 \pi^{2} r^{4}}(r-3 M) \ln \left(\frac{m}{\omega_{0}}\right)
$$

A physical realization of the Boulware vacuum would be the vacuum state outside a massive spherical body of radius only slightly larger than its Schwarzschild radius. According to

\footnotetext{
${ }^{1}$ It is worth pointing out that, in flat spacetime, the same result as Bethe's can be obtained if one employs a fully relativistic quantum field theoretic approach where no cutoff is present. See Refs. [17 20]
} 
Eq. (39), the Casimir-Polder-like force outside a massive spherical star can be either attractive or repulsive. In fact, the force is attractive close to the horizon and repulsive at the spatial asymptotic region with a behavior of $1 / r^{3}$. The turning point happens near $r \approx 3 M$ where the vacuum field modes are scattered the most. It is interesting to note that $r=3 M$ is the location of the unstable circular orbit of photons

\section{CASIMIR-POLDER-LIKE FORCE FOR UNRUH VACUUM}

For a static atom outside a black hole in interaction with fluctuating massless scalar fields in the Unruh vacuum, let us note that the field correlation function is given by [13, 14]

$$
G_{U}{ }^{+}\left(x, x^{\prime}\right)=\sum_{m l} \int_{-\infty}^{\infty} \frac{e^{-i \omega \Delta t}}{4 \pi \omega}\left|Y_{l m}(\theta, \phi)\right|^{2}\left[\frac{\left|\overrightarrow{R_{l}}(\omega, r)\right|^{2}}{1-e^{-2 \pi \omega / \kappa}}+\theta(\omega)\left|\overleftarrow{R_{l}}(\omega, r)\right|^{2}\right] d \omega
$$

where $\kappa=1 /(4 M)$ is the surface gravity of the black hole. The corresponding Fourier transform with the proper time $\tau$ of the two-level system reads

$$
\begin{aligned}
\mathcal{G}_{U}(\lambda) & =\int_{-\infty}^{\infty} e^{i \lambda \tau} G_{U}{ }^{+}\left(x, x^{\prime}\right) d \tau \\
& =\frac{1}{8 \pi \lambda} \sum_{l=0}^{\infty}\left[\theta\left(\lambda \sqrt{g_{00}}\right)(1+2 l)\left|\overleftarrow{R_{l}}\left(\lambda \sqrt{g_{00}}, r\right)\right|^{2}+\frac{(1+2 l)\left|\overrightarrow{R_{l}}\left(\lambda \sqrt{g_{00}}, r\right)\right|^{2}}{1-e^{-2 \pi \lambda \sqrt{g_{00}} / \kappa}}\right]
\end{aligned}
$$

A similar calculation as that in the Boulware vacuum yields the radiative energy-level shift of the ground-state in two special cases:

$$
\delta E_{-}^{U}=-i \mu^{2} \mathcal{K}_{U}\left(-\omega_{0}\right) \approx \begin{cases}\delta E_{0}+\delta E_{T}+\delta E_{0 r}, & (r \sim 2 M) \\ \delta E_{0}+\delta E_{T r}+\delta E_{0 r}, & (r \rightarrow \infty) .\end{cases}
$$

where

$$
\delta E_{T}=-\frac{\mu^{2} P}{2 \pi^{2}} \int_{0}^{\infty} \frac{\lambda}{1-e^{\lambda / T}}\left(\frac{1}{\lambda-\omega_{0}}-\frac{1}{\lambda+\omega_{0}}\right) d \lambda
$$

and

$$
\delta E_{T r}=-\frac{\mu^{2} P}{2 \pi^{2}} \int_{0}^{\infty} \frac{f(\lambda, r)}{1-e^{\lambda / T}}\left(\frac{\lambda}{\lambda-\omega_{0}}-\frac{\lambda}{\lambda+\omega_{0}}\right) d \lambda .
$$


Here, $T$ is given by

$$
T=\frac{\kappa_{r}}{2 \pi}=\frac{\kappa}{2 \pi \sqrt{g_{00}}}=\frac{T_{H}}{\sqrt{g_{00}}}
$$

with $T_{H}=\kappa / 2 \pi$, being the usual Hawking temperature of the black hole. This equation is the well-known Tolman relation [21, 22] which gives an effective temperature as measured by a local observer. A few comments are in order now. First, $\delta E_{T}$, which is a contribution to the energy-level shift of the atom close to the horizon, is structurally similar to the energylevel shift in a thermal heat bath at temperature $T$, while $\delta E_{T r}$, which is a contribution to that at infinity, shows the effect of backscattering of the thermal radiation off the spacetime curvature, represented by the grey-body factor $f(\lambda, r)$. These two terms clearly support the notion that there is a thermal radiation flux emanating from the black hole event horizon. Second, the other two terms, (i.e., $\delta E_{0}$ and $\left.\delta E_{0 r}\right)$ are just terms contained in $\delta E_{-}^{B}$.

To analyze the force on the atom in more detail, let us note that $\delta E_{T}$ can be approximated in the low- and high-temperature limits as follows

$$
\begin{aligned}
\delta E_{T} & =-\frac{\mu^{2} P}{2 \pi^{2}} \int_{0}^{\infty} \frac{\lambda}{1-e^{\lambda / T}}\left(\frac{1}{\lambda-\omega_{0}}-\frac{1}{\lambda+\omega_{0}}\right) d \lambda \\
& \approx \begin{cases}-\frac{\mu^{2} T^{2}}{6 \omega_{0}}-\frac{\mu^{2} \pi^{2} T^{4}}{15 \omega_{0}^{3}}, & \left(\omega_{0} \gg T\right) ; \\
\frac{\mu^{2} \omega_{0} \ln \left(\omega_{0}^{2} / T^{2}\right)}{2 \pi^{2}}, & \left(\omega_{0} \ll T\right) .\end{cases}
\end{aligned}
$$

According the Eq. (34), we can estimate $\delta E_{T r}$ in the same limits

$$
\delta E_{T r}=\frac{27 M^{2} g_{00}}{4 r^{2}} \delta E_{T} \approx \begin{cases}-\frac{9 \mu^{2} M^{2} T^{2} g_{00}}{8 \omega_{0} r^{2}}-\frac{9 \mu^{2} \pi^{2} M^{2} T^{4} g_{00}}{20 \omega_{0}^{3} r^{2}}, & \left(\omega_{0} \gg T\right) ; \\ \frac{27 \mu^{2} M^{2} \omega_{0} g_{00}}{8 \pi^{2} r^{2}} \ln \left(\frac{\omega_{0}^{2}}{T^{2}}\right), & \left(\omega_{0} \ll T\right) .\end{cases}
$$

Since close to the horizon, $g_{00}$ approaches zero, $T=T_{H} / \sqrt{g_{00}} \gg \omega_{0}$ is always satisfied. Consequently, the position-dependent energy-level shift can be written as

$$
\delta E_{-}^{U} \approx \frac{\mu^{2} \omega_{0}}{2 \pi^{2}} \ln \left[g_{00} \frac{\omega_{0}^{2}}{T_{H}^{2}}\right]+\frac{\mu^{2} \omega_{0}}{2 \pi^{2}} \ln \left(\frac{m}{\omega_{0}}\right)\left[1+\frac{27 M^{2} g_{00}}{4 r^{2}}\right] .
$$

When the atom is held static in the spatial asymptotic region, i.e., when $r \rightarrow \infty, T \sim T_{H}$. 
Now, the energy-level shift becomes

$$
\delta E_{-}^{U} \approx \begin{cases}-\frac{9 \mu^{2} M^{2} T_{H}^{2}}{8 \omega_{0}} \frac{1}{r^{2}}+\frac{\mu^{2} \omega_{0}}{2 \pi^{2}} \ln \left(\frac{m}{\omega_{0}}\right)\left[1+\frac{27 M^{2} g_{00}}{4 r^{2}}\right], & \left(\omega_{0} \gg T_{H}\right) ; \\ \frac{27 \mu^{2} M^{2} \omega_{0}}{8 \pi^{2} r^{2}} g_{00} \ln \left[g_{00} \frac{\omega_{0}^{2}}{T_{H}^{2}}\right]+\frac{\mu^{2} \omega_{0}}{2 \pi^{2}} \ln \left(\frac{m}{\omega_{0}}\right)\left[1+\frac{27 M^{2} g_{00}}{4 r^{2}}\right], & \left(\omega_{0} \ll T_{H}\right) .\end{cases}
$$

This position-dependent energy shift gives rise to a force on the atom which can be calculated by taking the first derivative with respect to $r$. Close to the horizon, we find that

$$
F^{U}=-\frac{\partial\left(\delta E_{-}^{U}\right)}{\partial r} \approx-\frac{\mu^{2} M \omega_{0}}{\pi^{2}(r-2 M) r}-\frac{27 \mu^{2} \omega_{0}}{64 M \pi^{2}} \ln \left(\frac{m}{\omega_{0}}\right)
$$

So the Casimir-Polder-like force is attractive and actually diverges at the event horizon. Let us note that the classical force that is needed to hold the atom static at the horizon also diverges. If the atom is in the spatial asymptotic region, this force can be approximated as

$$
F^{U}=-\frac{\partial\left(\delta E_{-}^{U}\right)}{\partial r} \approx \begin{cases}-\frac{9 \mu^{2} M^{2} T_{H}^{2}}{4 \omega_{0}} \frac{1}{r^{3}}+\frac{27 \mu^{2} M^{2} \omega_{0}}{4 \pi^{2}} \frac{1}{r^{3}} \ln \left(\frac{m}{\omega_{0}}\right), & \left(\omega_{0} \gg T_{H}\right) ; \\ -\frac{27 \mu^{2} M^{2} \omega_{0}}{2 \pi^{2}} \frac{1}{r^{3}} \ln \left(\frac{T_{H}}{\omega_{0}}\right)+\frac{27 \mu^{2} M^{2} \omega_{0}}{4 \pi^{2}} \frac{1}{r^{3}} \ln \left(\frac{m}{\omega_{0}}\right), \quad\left(\omega_{0} \ll T_{H}\right) .\end{cases}
$$

Typically, one has $m \gg \omega_{0}$, so, far from the black hole, the force will be attractive if $T_{H} \gg m$, and repulsive otherwise. It is interesting to note that the contribution to the force due to the presence of the Hawking radiation is always attractive. Therefore, the collapsing of a massive star into a black hole and the thermal radiation generated as a result makes the Casimir-Polder-like force more attractive than repulsive.

\section{CONCLUSION}

In summary, we have calculated the energy-level shift of a two-level atom outside a spherically symmetric black hole in the paradigm of open quantum systems by looking at the time evolution of the atom interacting with massless scalar fields in the Boulware vacuum and the Unruh vacuum. The time evolution of the atom is governed by a master equation 
obtained by tracing over the field degrees of freedom from the complete system. Our results show that, for an atom in the ground state, the level shift is position-dependent and gives rise to a force on the atom besides the classical gravitational force.

For the case of the Boulware vacuum, which represents a star that has not collapsed through its event horizon, this force is attractive near the horizon, and is repulsive far away from the black hole with a behavior of $r^{-3}$. The turning point occurs near $r \sim 3 M$ where

the vacuum field modes are scattered the most. For the case of the Unruh vacuum which represents a radiating black hole, we find that the contribution to the Casimir-Polder-like force due to the presence of Hawking radiation is always attractive, and in fact this attractive force diverges as the horizon is approached.

\section{Acknowledgments}

One of us (HY) would like to thank the Kavli Institute for Theoretical Physics China where part of this work was done. This work was supported in part by the National Natural Science Foundation of China under Grants No. 11075083, No.11005038 and No. 10935013; the Zhejiang Provincial Natural Science Foundation of China under Grant No. Z6100077; the National Basic Research Program of China under Grant No. 2010CB832803; the PCSIRT under Grant No. IRT0964; the Hunan Provincial Natural Science Foundation of China under Grant No. 11JJ7001; and the Program for the Key Discipline in Hunan Province.

[1] D. Meschede, W. Jhe and E.A. Hinds, Phys. Rev. A 41, 1587 (1990).

[2] J. Audretsch and R. Müller, Phys. Rev. A 52, 629 (1995).

[3] R. Passante, Phys. Rev. A 57, 1590 (1998).

[4] L. Rizzuto, Phys. Rev. A 76, 062114 (2007).

[5] Z. Zhu and H. Yu, Phys. Rev. A, 82, 042108 (2010).

[6] H.-P. Breuer and F. Petruccione, The Theory of Open Quantum Systems (Oxford University Press, Oxford, 2002). 
[7] H. Yu and J. Zhang, Phys. Rev. D 77, 024031 (2008); Proceedings of the Ninth Asia-Pacific International Conference on Gravitation and Astrophysics, Wuhan, China, 2009, edited by J. Luo et al (World Scientific Publishing, Singpore, 2010), p. 319.

[8] H. Yu, Phys. Rev. Lett. 106, 061101 (2011).

[9] F. Benatti, R. Floreanini and M. Piani, Phys. Rev. Lett. 91, 070402 (2003).

[10] F. Benatti and R. Floreanini, Phys. Rev. A 70, 012112 (2004).

[11] F. Benatti and R. Floreanini, J. Opt. B: Quantum Semiclass. Opt. 7,S429-S434 (2005).

[12] V. Gorini, A. Kossakowski, and E. C. G. Surdarshan, J. Math. Phys. 17, 821 (1976); G. Lindblad, Commun. Math. Phys. 48, 119 (1976).

[13] N. D. Birrell and P. C. W. Davies , Quantum Fields in Curved Space(Cambridge University Press,Cambridge,1982).

[14] P. Candelas, Phys. Rev. D 21, 2185(1980).

[15] B.S. Dewitt, Physics Reports 19, 295 (1975).

[16] H.A. Bethe, Phys. Rev. 72, 339 (1947).

[17] P.W. Milonni, The Quantum Vacuum: An Introduction to Quantum Electrodynamics (Academic Press, San Diego, 1994), p. 86.

[18] W. Greiner and J. Reinhart,Quantum Electrodynamics (3rd edition, Springer- Verlag, Berlin Heidelberg New York,2003), p. 332.

[19] N. M. Kroll and W. E. Lamb, Phys. Rev. 75, 388 (1949).

[20] J. B. French and V. F. Weisskopf, Phys. Rev. 75, 1240 (1949).

[21] R. Tolman, Phys. Rev. 35, 904 (1930).

[22] R. Tolman and P. Ehrenfest, Phys. Rev. 36, 1791 (1930). 